\title{
Final design and development status of the acquisition and guiding system for SOXS.
}

Anna Brucalassi*,a,b, Giuliano Pignata ${ }^{\mathrm{a}, \mathrm{c}}$, José Antonio Araiza-Duran ${ }^{\mathrm{c}, \mathrm{v}}$, Sergio Campana ${ }^{\mathrm{d}}$, Riccardo Claudie, Pietro Schipani ${ }^{\mathrm{f}}$, Matteo Aliverti ${ }^{\mathrm{d}}$, Andrea Baruffolo ${ }^{\mathrm{e}}$, Sagi Ben-Ami ${ }^{\mathrm{g}}$, Federico Biondi $^{\mathrm{h}}$, Giulio Capasso ${ }^{\mathrm{f}}$, Mirko Colapietro ${ }^{\mathrm{f}}$, Rosario Cosentino ${ }^{\mathrm{i}}$, Francesco D'Alessio ${ }^{\mathrm{j}}$, Paolo D'Avanzo d, Matteo Genoni ${ }^{\mathrm{d}}$, Ofir Hershkok ${ }^{\mathrm{k}}$, Hanindyo Kuncarayakti ${ }^{\mathrm{l}, \mathrm{m}}$, Marco Landoni ${ }^{\mathrm{d}}$, Matteo Munari ${ }^{\mathrm{n}}$, Kalian Radhakrishnan ${ }^{\mathrm{e}}$, Michael Rappaport ${ }^{\mathrm{k}}$, Davide Ricci ${ }^{\mathrm{e}}$, Adam Rubin ${ }^{\circ}$, Salvatore Scuderi ${ }^{\mathrm{p}}$, Fabrizio Vitali ${ }^{\mathrm{j}}$, Ricardo Zanmar Sanchez ${ }^{\mathrm{n}}$, David Young ${ }^{\mathrm{q}}$, Jani Achrén ${ }^{\mathrm{r}}$, Iair Arcavis ${ }^{\mathrm{s}}$, Rachel Bruch ${ }^{\mathrm{k}}$, Enrico Cappellaro ${ }^{\mathrm{e}}$, Massimo Della Valle ${ }^{\mathrm{f}}$, Marco De Pascale ${ }^{\mathrm{e}}$, Rosario Di Benedetton ${ }^{n}$, Sergio D’Orsi ${ }^{\mathrm{f}}$, Avishay Gal-Yam ${ }^{\mathrm{k}}$, Marcos Hernandez ${ }^{\mathrm{i}}$, Jari Kotilainen ${ }^{\mathrm{l}, \mathrm{m}}$, Gianluca Li Causi ${ }^{\mathrm{t}}$, Seppo Mattila ${ }^{\mathrm{m}}$, Marco Riva ${ }^{\mathrm{d}}$, Bernardo Salasnich ${ }^{\mathrm{e}}$, Stephen Smartt ${ }^{\mathrm{q}}$, Maximilian Stritzinger ${ }^{\mathrm{u}}$, and Hector Ventura ${ }^{\mathrm{i}}$

${ }^{a}$ Universidad Andres Bello, Avda. Republica 252, Santiago, Chile

${ }^{b}$ INAF - Osservatorio Astronomico di Arcetri, Largo Fermi 5, I-50125, Florence, Italy

${ }^{\mathrm{c}}$ Millennium Institute of Astrophysics (MAS)

${ }^{\mathrm{d}} \mathrm{INAF}$ - Osservatorio Astronomico di Brera, Via Bianchi 46, I-23807, Merate, Italy

'INAF - Osservatorio Astronomico di Padova, Vicolo dell'Osservatorio 5, I-35122, Padua, Italy ${ }^{\mathrm{f}}$ INAF - Osservatorio Astronomico di Capodimonte, Sal. Moiariello 16, I-80131, Naples, Italy 'Harvard-Smithsonian Center for Astrophysics, Cambridge, USA

hMax-Planck-Institut für Extraterrestrische Physik, Giessenbachstr. 1, D-85748 Garching, Germany

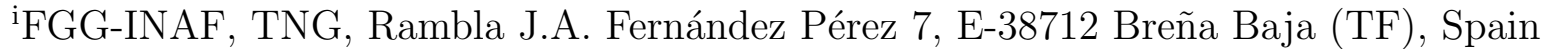
jINAF - Osservatorio Astronomico di Roma, Via Frascati 33, I-00078 M. Porzio Catone, Italy ${ }^{k}$ Weizmann Institute of Science, Herzl St 234, Rehovot, 7610001, Israel

${ }^{1}$ Finnish Centre for Astronomy with ESO (FINCA), FI-20014 University of Turku, Finland mTuorla Observatory, Dept. of Physics and Astronomy, FI-20014 University of Turku, Finland

${ }^{n}$ INAF - Osservatorio Astrofisico di Catania, Via S. Sofia 78 30, I-95123 Catania, Italy ${ }^{\circ}$ European Southern Observatory, Karl Schwarzschild Str. 2, D-85748, Garching bei München PINAF - Istituto di Astrofisica Spaziale e Fisica Cosmica,Via Corti 12, I-20133 Milan, Italy 'Queen's University Belfast, Belfast, County Antrim, BT7 1NN, UK ${ }^{r}$ Incident Angle Oy, Capsiankatu 4 A 29, FI-20320 Turku, Finland sTel Aviv University, Department of Astrophysics, 69978 Tel Aviv, Israel ${ }^{\mathrm{t}} \mathrm{INAF}$ - Istituto di Astrofisica e Planetologia Spaziali, Rome, Italy

"Aarhus University, Ny Munkegade 120, D-8000 Aarhus, Denmark

${ }^{v}$ Centro de Investigaciones en Optica A. C., Loma del Bosque 115, Lomas del Campestre, 37150 Leon Guanajuato, Mexico

\section{ABSTRACT}

SOXS (Son Of X-Shooter) will be the new medium resolution ( $\mathrm{R} \sim 4500$ for $1^{\prime \prime}$ slit), high-efficiency, wide band spectrograph for the ESO NTT at La Silla, optimized for classification and follow-up of transient events. SOXS

\footnotetext{
${ }^{*}$ Contact information: A.B: anna.brucalassi@gmail.com, Telephone: +39 0552752243
}

G.P: pignata@gmail.com, Telephone: +33 (0)1 98765432 
will simultaneously cover UV-optical and NIR bands (0.35-2.00 micron) using two different arms and a pre-slit Common Path feeding system. The instrument will be also equipped by a Calibration Unit and an Acquisition Camera (AC) System. In this paper we present the final opto-mechanical design for the AC System and we describe its development status. The project is currently in manufacturing and integration phases.

Keywords: SOXS, Spectroscopy, Imaging, Acquisition and Guiding

\section{INTRODUCTION}

The Son Of X-Shooter (SOXS), is the new instrument, ${ }^{1,2}$ actually under manufacturing and integration phase, for the European Southern Observatory (ESO) New Technologies Telescope (NTT) at the La Silla Observatory, Chile. It will be dedicated to the follow up of any kind of transient events ensuring fast response time, high efficiency and availability. SOXS will simultaneously cover the electromagnetic spectrum from 0.35 to $2.0 \mu \mathrm{m}$ with a spectral resolution of R 4500, using two arms (UV-VIS and NIR) $)^{3,4}$ and a pre-slit Common Path (CP) feeding system. ${ }^{5,6}$

SOXS is also equipped with a Calibration sub-unit and an Acquisition Camera system (CAM $)^{7}$ attached directly to the CP. The CAM system is foreseen to work not only for target acquisition and (optional) secondary guiding, but it will be also equipped with a filter wheel and a scientific camera for providing photometric observations.

The schedule for the development of the CAM system has been seriously affected by the ongoing COVID19 pandemic, interrupting some providers activities and resulting in a delay of about some months. In the following we summarize the final opto-mechanical design for the AC System and we describe its development status.

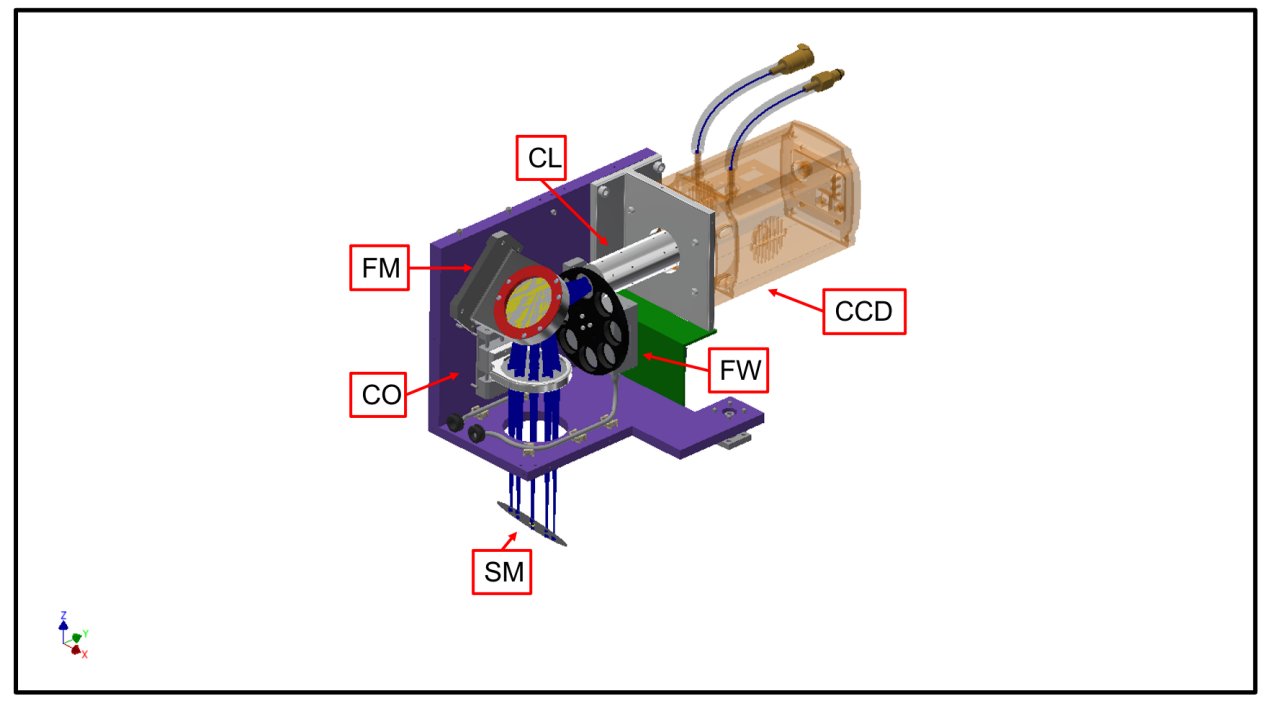

Figure 1. Overview of the Acquisition Camera System: it consists of a collimator lens (CO), folding mirror (FM), filter wheel $(\mathrm{FW})$, focal reducer optics (CL) and CCD camera. All the elements are included in a structure made of 6061-T6 Aluminum.

\section{OVERVIEW}

Figure 1 shows an overview of the Acquisition Camera System. It is based on a collimator lens (CO), a folding mirror (FM), a filter wheel (FW) with a broad-band filter set (ugrizY and V-Johnson), a focal reducer optics (CL) and CCD camera. All the system is included in a structure made of 6061-T6 Aluminum. The detector is

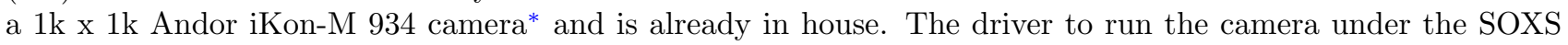
control software has been developed and validated. ${ }^{8,9}$

\footnotetext{
${ }^{*}$ https://andor.oxinst.com
} 
A system named as CAM selector and placed in the CP at the level of of the Nasmyth focal plane will redirect the F/11 beam from the telescope toward the Acquisition and Camera system.

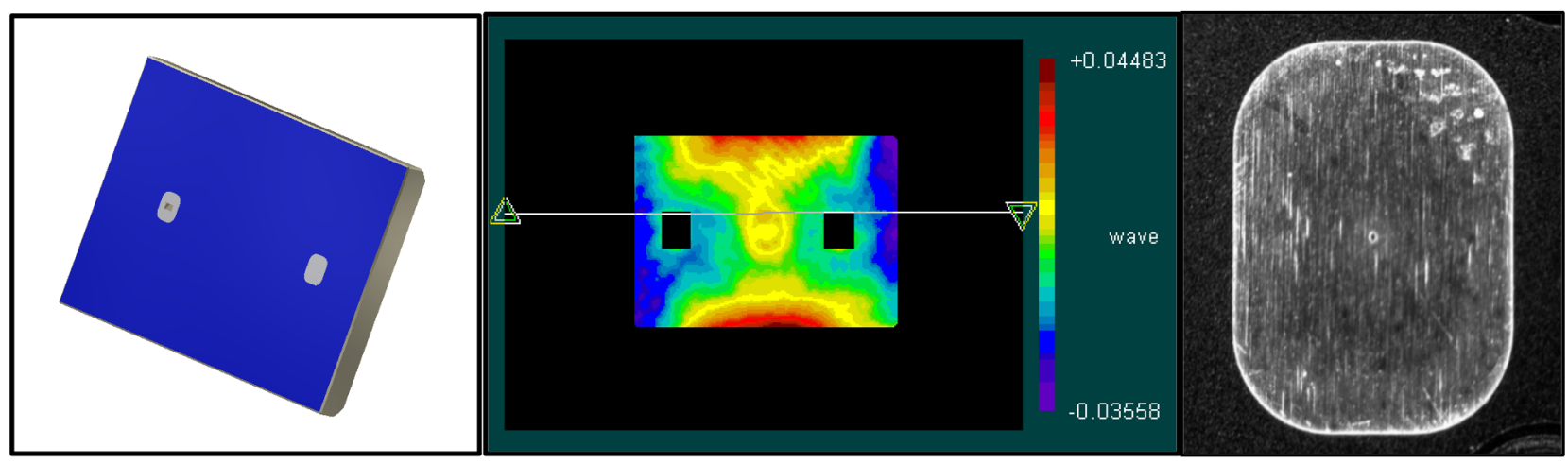

Figure 2. Left panel: view of the selector mirror. Central pannel: Wavefront Map of the selector mirror: PV $=0.161$ fr, $\mathrm{rms}=0.014$ wave . Right panel: zoomed view of the metallic plate for the Artificial Star Mode.

The CAM selector system consists of a linear stage that carries a pellicle beam-splitter and a single mirror with three positions for different functions (Acquisition and Imaging, Artificial Star and Spectroscopy). The mirror and the pellicle beam-splitter are tilted at $45^{\circ}$ and direct light from sky or from the slits, respectively, to the CAM optics. The pellicle beam-splitter allows us to use the CAM system as slit viewing camera (with the calibration lamp on). In Acquisition and Imaging Mode, the selector mirror redirects the full field towards the CCD and the CAM system can be operated for photometric observations. With the selecor mirror in Artificial Star Mode a 0.5 arcsec reference pinhole acts as an artificial star on the focal plane by switching on the calibration lamp. Finally, in Spectroscopy Mode, the selector mirror passes an unvignetted field of 15 arcsec to the spectrograph slits, whereas the peripheral field is simultaneously imaged on the camera. Thus, off-axis secondary guiding is possible using peripheral sources. We refer to 7,10 for a more detailed description of the CAM selector system and its functionalities.

The selector mirror has been already manufactured and is based on a rectangular Fused Silica mirror (91.0x70.0x10.0mm) with two holes that allow the optical beam to pass. On the reflective surface two specific engravings ensure two corresponding metallic plates to be fixed by glue. On the center of the metallic plates a 0.5 arcsec reference pinhole and a rectangular slit-hole for a 15 arsec unvignetted beam have been created by laser cut. Figure 2 shows a view of the selector mirror (left panel), its Wavefront Map (central panel) and a zoomed picture of the metallic plate for the Artificial Star Mode (right panel).

\section{FINAL OPTICAL DESIGN}

After the Final Design Review, the CAM optical design needed some modifications due to the unfeasible waiting time for the procurement of some glasses. However, the general architecture and concept of the design has been preserved. The new optical design is illustrated in Figure 3 and in Table 1.

The first element after the CAM selector mirror is a collimator lens of $65 \mathrm{~mm}$ diameter that reimages the pupil onto a compact camera. A folding mirror and a filter wheel, illustrated in Figure 3 by a single plane glass, follow in the optical path. The subsequent camera lens, relays the Nasmyth focus on the detector, with an $F_{\#}=3.6$, through 2 doublets and two singlets of max diameter $30.0 \mathrm{~mm}$. A Field of View of $3.5 \times 3.5$ arcmin (linear) can be reached in imaging mode, resulting in a pixel scale of $\sim 0.2$ arcsec.

The total length from focus to focus is $430.46 \mathrm{~mm}$.

\subsection{Image Quality and Thermal Analysis}

Figure 4 shows the spot diagrams and the geometric encircled energy for the new optical configuration. The design has been done considering $10^{\circ} \mathrm{C}$ and 0.76 atm. Plotted field positions correspond to the center of the field, sides of the detector in $\mathrm{x}$ and $\mathrm{y}$ at 3.5 arcmin and the position along the diagonal corresponding to a 3.75 


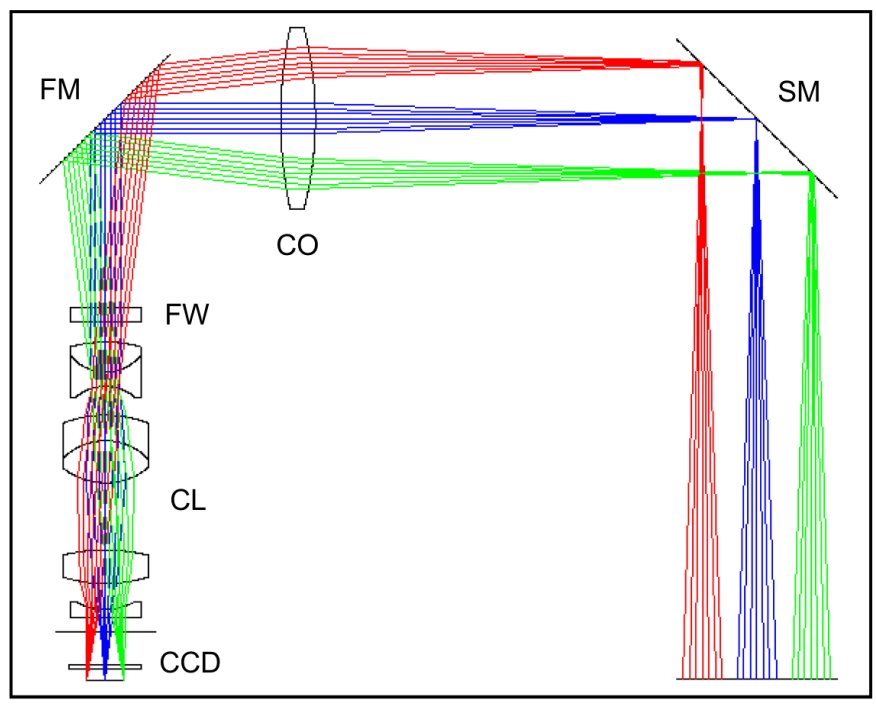

Figure 3. Optical Layout of the CAM System. From right to the left: the Selector Mirror (SM), the Collimator lens (CO), the Folding Mirror (FM), the Filter Wheel (FW), the Camera Lens (CL) with two doublets (D1, D2), the two singlets (S1, S2), the detector window and the focal plane.

arcmin diameter, for which the quality is still good. Clearly, the spot is contained in a box of 2x2 pixels (26.0 $\mu \mathrm{m})$ for all the analyzed views.
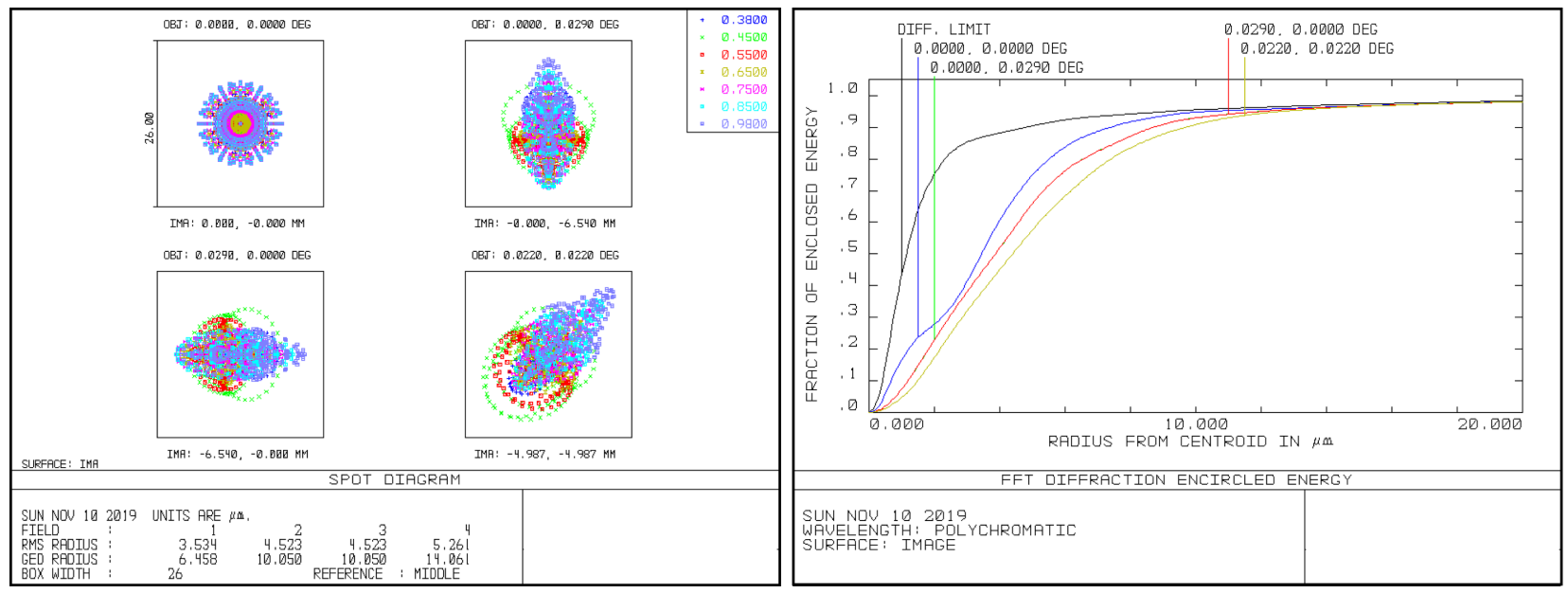

Figure 4. Left panel: CAM Spot diagrams for field positions correspond to on-axis, 3.5 arcmin (side of the detector) in $\mathrm{x}$ and $\mathrm{y}$ direction and along the diagonal corresponding to a 3.75 arcmin diameter (corner of the detector). Right panel: Geometric encircled energy.

Figure 5 instead presents the spot diagrams of the new optical configuration considering temperatures of $0^{\circ}$ $\mathrm{C}$ (left panel) and $20^{\circ} \mathrm{C}$ (right panel). A change of $\pm 10^{\circ} \mathrm{C}$ causes a deterioration of the image, however, always with a geometrical dimension of about 2 pixels $(26.0 \mu \mathrm{m})$. In addition, the optical quality can be completely recovered re-focusing with the collimator lens.

\section{DEVELOPMENT STATUS}

At the time of writing, all the optical parts together with their opto-mechanical mounts are in production phase. The external structure that will host the CAM system will be made by Aluminum 6061-T6 with a T-Shape as 
Table 1. Optical Elements data.

\begin{tabular}{|l|l|l|l|l|}
\hline Description & Radius $(\mathrm{mm})$ & Distance $(\mathrm{mm})$ & Material & Diameter $(\mathrm{mm})$ \\
\hline Telescope Focus & - & 155.81 & - & - \\
\hline Collimator & 132.10 & 12.24 & N-FK58 & 65.00 \\
\hline & -158.48 & 61.84 & - & 65.00 \\
\hline Folding Mirror & - & -67.37 & - & 65.00 \\
\hline Filter & - & -5.00 & N-BK7 & 22.00 \\
\hline & & -7.38 & - & 22.00 \\
\hline D1 & -44.91 & -10.74 & N-FK58 & 25.00 \\
\hline & 16.01 & -5.09 & K10 & 25.00 \\
\hline & -15.97 & -10.27 & - & 21.00 \\
\hline D2 & -40.98 & -9.11 & K10 & 30.00 \\
\hline & -20.54 & -15.02 & N-FK58 & 30.00 \\
\hline & 23.98 & -24.00 & - & 30.00 \\
\hline S1 & -62.09 & -12.00 & LLF1 & 30.00 \\
\hline & 48.73 & -9.19 & - & 30.00 \\
\hline S2 & 21.25 & -2.97 & PBL6Y & 21.00 \\
\hline & - & -4.93 & - & 25.00 \\
\hline Shutter & - & -11.85 & - & - \\
\hline Detector Window & - & -1.50 & SILICA & 25.40 \\
\hline & - & -4.15 & - & 25.40 \\
\hline Focal Plane & - & & & - \\
\hline
\end{tabular}

shown in Figure 1. All the walls are structural with thickness of $\sim 15 \mathrm{~mm}$, while the cover is not structural and is made of a $3 \mathrm{~mm}$ thick Aluminum plate.

The detector will be mounted on a support directly connected to the external structure. The centering of the CCD will be done shimming the 3 holes and 3 dowel pins used to place the CCD together with the support system.

\subsection{Collimator Lens}

The $65 \mathrm{~mm}$ diameter collimator lens is the first element after the CAM selector mirror along the optical path. It will be placed on a linear stage with $15 \mathrm{~mm}$ stroke (PI M-111.1DG1) and it will act as a re-focuser. The linear stage is in house and already controlled. The lens is glued to an Aluminum mount by $33 \mathrm{M} 2216$ glue spots (see Figure 6, left panel).

\subsection{Folding Mirror}

Figure 7 shows the fused silica folding mirror with its opto-mechanical mount (left side), that is at the moment under manufacturing. The mirror mount will be screwed on the front surface of the external CAM structure. The mirror will be kept in position inside the mount frame by a styrodur ring and a support. The beam is redirected through the folding mirror along an axis parallel to the main telescope axis.

\subsection{Filter Wheel}

In front of the camera lens there is a filter wheel made by a rotary stage (PI M-116.DG) and a custom filter support, which can host up to 8 elements. A broad-band filter set (ugriz $Y$ and $V$-Johnson) from Asahi Spectra 

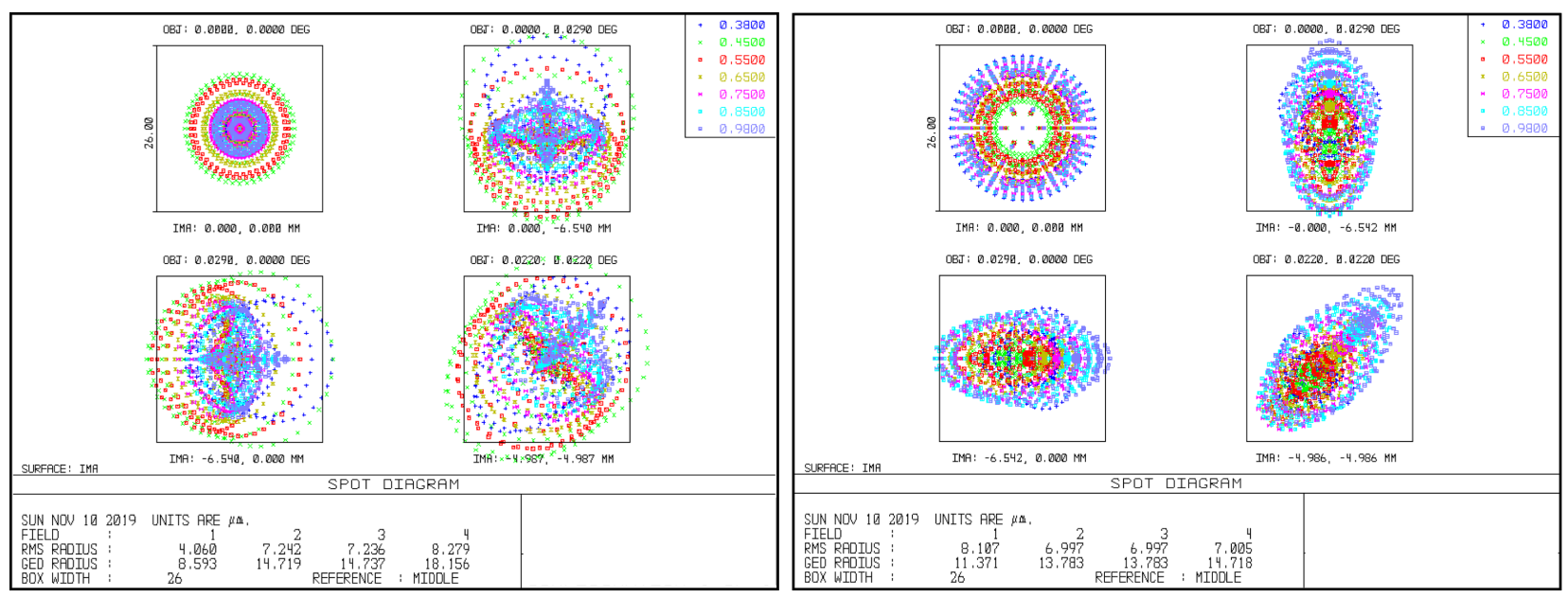

Figure 5. Spot diagrams considering temperatures of $0^{\circ} \mathrm{C}$ (left panel) and $20^{\circ} \mathrm{C}$ (right panel). The geometrical dimension of the spot are still about 2 pixels $(26.0 \mu \mathrm{m})$.
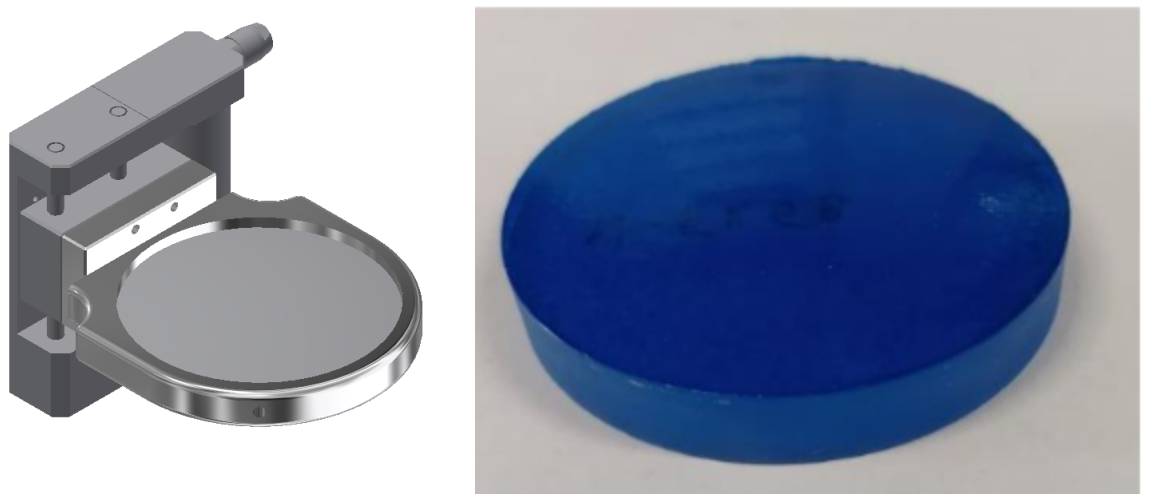

Figure 6. Left panel: view of the opto-mechanical mount for the collimator lens. Right panel: image of the lens under manufacturing.

$\mathrm{USA}^{\dagger}$ are already in house. Figure 8 shows the filter transmission as function of wavelength as tested after the production. The filter selection has been optimized to match as best as possible the LSST filter passbands. A position of the filter wheel is left empty for the Spectroscopy mode.

\subsection{Camera lens}

The 2 doublets and 2 singlets of max diameter of $30 \mathrm{~mm}$ forming the camera lens are under construction. The camera lens will relay the Nasmyth focus on the detector, with a $\mathrm{F}_{\#}=3.6$. It is planned to include the 2 doublets and the 2 singlets in a tubular structure anchored to the CCD mounting. The optical components will be holded inside the tube by specific spacers and creating $33 \mathrm{M} 2216$ glued spots on the side of the element. This operation will be performed via small holes radially drilled inside the support.

\section{CONCLUSIONS}

The development of the CAM system has been seriously affected by the ongoing COVID19 pandemic, interrupting some providers activities and resulting in a delay of about some months. A new optical design has been development, keeping the general design concept, to mitigate the unfeasible waiting time for the procurement of some glasses. However, all the CAM parts are in an advanced phase of realization, very close to be completed.

\footnotetext{
${ }^{\dagger}$ https://www.asahi-spectra.com/
} 

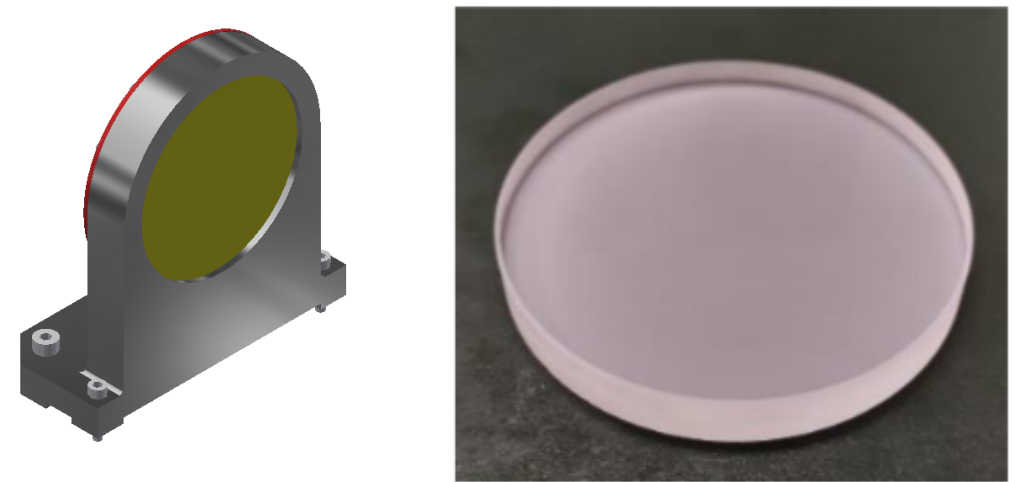

Figure 7. Left panel: view of the opto-mechanical mount for the folding mirror. Right panel: image of the fused silica part under manufacturing.
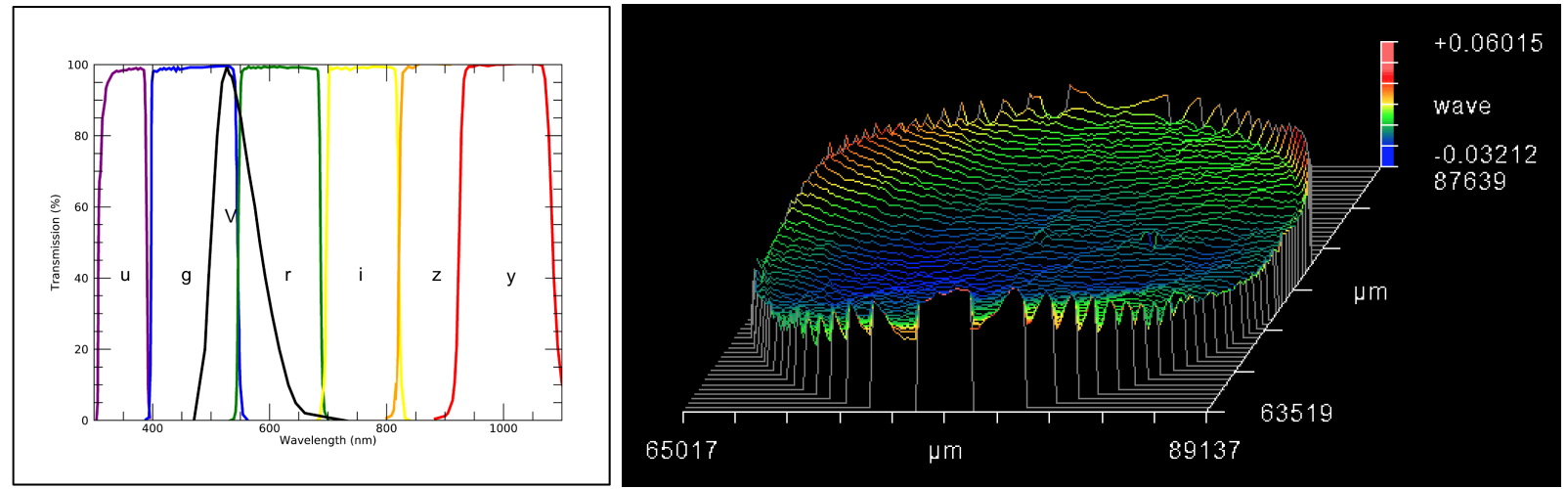

Figure 8. Left: Transmission of the CAM broad-band filter set as function of wavelength as tested after the production. The filter selection has been optimized to follow the LSST filter passbands. Right: Wavefront Map for the r-band filter. $\mathrm{PV}=0.092$ wave, $\mathrm{rms}=0.011$ wave.

The CAM system will be assembled in Italy (INAF-Padua) where also the preliminary integration and final test for the complete instrument SOXS will be done before the shipment to the NTT in La Silla. The instrument integration and test phase is planned in 2021.

\section{REFERENCES}

[1] Schipani, P., Campana, S., et al., "SOXS: a wide band spectrograph to follow up transients," in [Groundbased and Airborne Instrumentation for Astronomy VII], Evans, C. J., Simard, L., and Takami, H., eds., Society of Photo-Optical Instrumentation Engineers (SPIE) Conference Series 10702, 107020F (July 2018).

[2] Schipani, P., Claudi, R., et al., "The new SOXS instrument for the ESO NTT," in [Ground-based and Airborne Instrumentation for Astronomy VI], Proc. SPIE 9908, 990841 (Aug. 2016).

[3] Rubin, A., Ben-Ami, S., et al., "MITS: the multi-imaging transient spectrograph for SOXS," in [Groundbased and Airborne Instrumentation for Astronomy VII], Evans, C. J., Simard, L., and Takami, H., eds., Society of Photo-Optical Instrumentation Engineers (SPIE) Conference Series 10702, 107022Z (July 2018).

[4] Zanmar Sanchez, R., Munari, M., et al., "Optical design of the SOXS spectrograph for ESO NTT," in [Ground-based and Airborne Instrumentation for Astronomy VII], Evans, C. J., Simard, L., and Takami, H., eds., Society of Photo-Optical Instrumentation Engineers (SPIE) Conference Series 10702, 1070227 (July 2018).

[5] Claudi, R., Aliverti, M., et al., "The common path of SOXS (Son of X-Shooter)," in [Ground-based and Airborne Instrumentation for Astronomy VII], Evans, C. J., Simard, L., and Takami, H., eds., Society of Photo-Optical Instrumentation Engineers (SPIE) Conference Series 10702, 107023T (July 2018). 
[6] Biondi, F., Claudi, R., et al., "The assembly integration and test activities for the new SOXS instrument at NTT," in [Ground-based and Airborne Instrumentation for Astronomy VII], Evans, C. J., Simard, L., and Takami, H., eds., Society of Photo-Optical Instrumentation Engineers (SPIE) Conference Series 10702, 107023D (July 2018).

[7] Brucalassi, A., Araiza-Durán, J. A., Pignata, G., et al., "The acquisition camera system for SOXS at NTT," in [Ground-based and Airborne Instrumentation for Astronomy VII], Evans, C. J., Simard, L., and Takami, H., eds., Society of Photo-Optical Instrumentation Engineers (SPIE) Conference Series 10702, 107022M (July 2018).

[8] Capasso, G., Colapietro, M., et al., "SOXS control electronics design," in [Software and Cyberinfrastructure for Astronomy V], Guzman, J. C. and Ibsen, J., eds., Society of Photo-Optical Instrumentation Engineers (SPIE) Conference Series 10707, 107072H (July 2018).

[9] Ricci, D., Baruffolo, A., et al., "Architecture of the SOXS instrument control software," in [Software and Cyberinfrastructure for Astronomy V], Guzman, J. C. and Ibsen, J., eds., Society of Photo-Optical Instrumentation Engineers (SPIE) Conference Series 10707, 107071G (July 2018).

[10] Aliverti, M., Hershko, O., et al., "The mechanical design of SOXS for the NTT," in [Ground-based and Airborne Instrumentation for Astronomy VII], Evans, C. J., Simard, L., and Takami, H., eds., Society of Photo-Optical Instrumentation Engineers (SPIE) Conference Series 10702, 1070231 (July 2018). 